\title{
Research on the Safe Distance of UAV Carrying Manipulator for Inspection and Repair of Transmission Line
}

\author{
W L Zhang ${ }^{1}$, W Y Wang ${ }^{2}$, G Y Ji ${ }^{1}, \mathrm{H} \mathrm{Shi}^{2}, \mathrm{~J} \mathrm{Pan}^{3}, \mathrm{P} \mathrm{J} \mathrm{Niu}^{4}$ and G Q Su${ }^{1}$ \\ ${ }^{1}$ State Grid Tianjin Electric Power Company Chengnan Power Supply Branch, Tianjin, Tianjin, 300000, China \\ ${ }^{2}$ School of Electrical Engineering and Automation, Tianjin Polytechnic University, Tianjin, Tianjin, 300000, China \\ ${ }^{3}$ School of Electronics and Information Engineering, Tianjin Polytechnic University, Tianjin, Tianjin, 300000, China \\ ${ }^{4}$ Tianjin Polytechnic University, Tianjin, Tianjin, 300000, China
}

\begin{abstract}
When the drone is equipped with a robotic arm to replace the manual maintenance of the transmission line, the insulating parts in the drone maintenance system are prone to discharge and breakdown risks. In this paper, combined with the actual parameters of the transmission line and the UAV maintenance system, the finite element software is used to establish the electric field simulation model of the transmission line and the UAV maintenance system, and mainly analyzes the influence of the electric field around the transmission line on the UAV maintenance system at different distances. It is concluded that the distance between the overhaul system of the drone equipped with a robotic arm is greater than or equal to $0.02 \mathrm{~m}$, and there is no discharge breakdown of the insulation components; and the drone is carried out by building a test platform for the drone equipped with robotic arm to overhaul the transmission line. The safety distance test study of the robotic arm overhaul system shows that the safe working distance of the insulated components of the drone equipped with robotic arm overhaul system is $0.09 \mathrm{~m}$. This research provides a guarantee for the safe operation of the power transmission line equipped with the robotic arm overhauled by the drone in actual scenarios.
\end{abstract}

\section{Introduction}

The reliability, stability and power supply quality of electric energy play a vital role in national economic development [1]. my country's transmission lines cover a wide range and the terrain is complex, which poses greater challenges for its normal operation, inspection and maintenance. The traditional manual maintenance method has high intensity, long cycle and low efficiency, and it is difficult to carry out maintenance work in some areas with complex terrain or even dangerous. Compared with the traditional maintenance methods, the maintenance system of the UAV equipped with the robotic arm has the advantages of high efficiency, good operability and flexibility, less restricted by the terrain, and reduced casualties [2][3].

At present, many scholars have carried out research on the transmission line inspection system based on UAV. Through reading the literature, literature [4][5] mainly studies the safety distance measurement method in the patrol inspection of UAV transmission lines. Literature [6]-[9] mainly studies the image recognition and fault location functions of UAV inspection systems. Literature [10][11] mainly studies the impact of UAVs on the electric field around transmission lines and the safety distance between UAV inspection systems and transmission lines. Literature [12][13] mainly studies the automatic obstacle avoidance technology of UAV in the process of transmission line inspection. With the development of unmanned aerial vehicle technology, the concept of unmanned aerial vehicle equipped with a robotic arm has been proposed. Therefore, there are relatively few researches on the safety distance of the unmanned aerial vehicle equipped with robotic arm to repair power transmission lines

In this paper, the electric field simulation model is established based on the $35 \mathrm{kV}$ transmission line and the UAV maintenance system. Under the normal operation of the transmission line, the influence of the electric field around the transmission line on the UAV-mounted robotic arm maintenance system and the maximum electric field strength and the voltage difference between the two ends of the UAV maintenance system are analyzed. Then, a test platform for the safe distance of power transmission lines with drones equipped with robotic arms was built, and the minimum safe distances for power transmission lines with drones equipped with robotic arms were obtained.

\section{Method}

In this paper, finite element software is used to establish an electric field simulation model. Through simulation, the maximum electric field strength of each component and the difference between the left and right voltages of each component are obtained during the operation of the 
maintenance system of the UAV equipped manipulator arm. The $35 \mathrm{kV}$ transmission line designed by Tianjin Electric Power Company is shown in figure 1(a). According to the tower design literature provided by Tianjin Electric Power Company, the tower has a total height of $31200 \mathrm{~mm}$, a large umbrella radius of $2400 \mathrm{~mm}$, a medium umbrella radius of $3200 \mathrm{~mm}$, and a small umbrella radius. It is $2700 \mathrm{~mm}$, and the transmission line wire adopts LGJ-150 model with a diameter of $17.48 \mathrm{~mm}$.

The maintenance system of the drone equipped with a robotic arm is built based on the inspection drone system. The robotic arm is made of epoxy resin, and the drone's rotor, frame, and body are all made of carbon fiber materials. As shown in figure 1(b).

Figure 2 shows the establishment of a two-dimensional simulation model for the overhaul of transmission lines with a drone equipped with a robotic arm. The distance from the left end of the mechanical arm to the geometric center of the line is set as $d$, and the phase voltage of a certain phase of the transmission line is $35 / \sqrt{ } 3 \mathrm{kV}$. The main insulation components in the overhaul system of the drone equipped with the robotic arm are: the robotic arm, the frame, the fuselage, the left rotor, and the right rotor, as shown in figure 2.

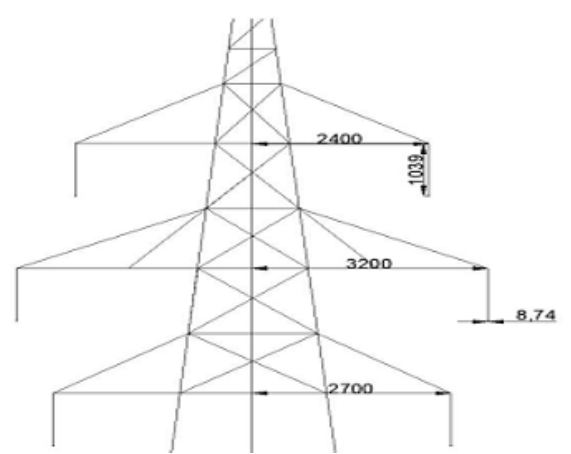

(a)

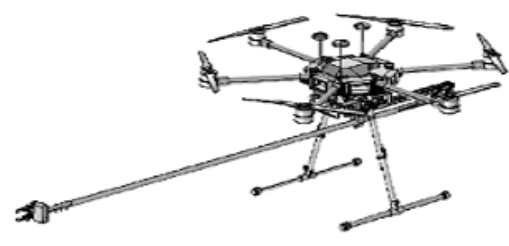

(b)

Fig1. (a) transmission line tower's floor plan (b) based on arm unmanned aircraft maintenance system

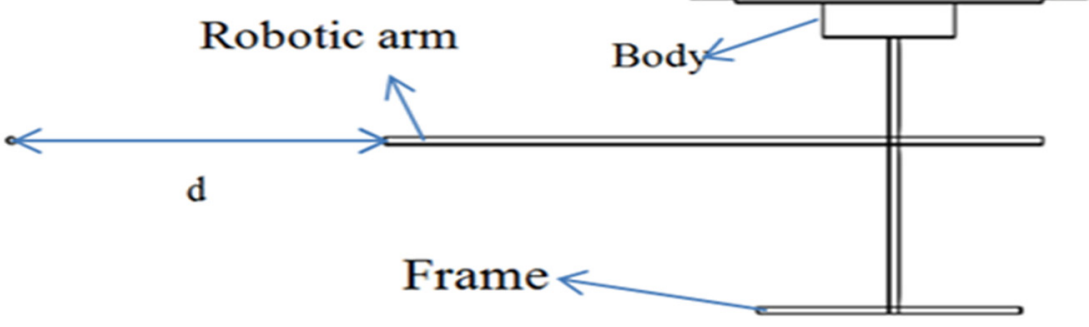

Fig2. Simulation model diagram

\section{Simulation analysis of the maximum electric field intensity borne by insulating parts}

In the simulation, a $35 / \sqrt{ } 3 \mathrm{kV}$ voltage is applied to the wire, and the electric field intensity distribution around the transmission line is shown in figure $3(\mathrm{~b})$, which is distributed in a multi-layer circular ring, and the electric field intensity gradually weakens from the inside to the outside.

During the work process of the maintenance system of the drone equipped with a robotic arm, the electric field around the transmission line will affect it. Therefore, the simulation model of the drone equipped with the robotic arm is placed in figure 3(b), and the drone equipped with the robotic arm is analyzed separately When the distance between the inspection system and the transmission line is $\mathrm{d}=0.84 \mathrm{~m}, \mathrm{~d}=0.34 \mathrm{~m}$, and $\mathrm{d}=0.02 \mathrm{~m}$, the maximum electric field strength that each insulating component of the UAV inspection system will bear and the voltage difference between the two ends of each insulating component. 


\subsection{The distance from the power transmission line $d=0.84 \mathrm{~m}$ from the overhaul system of the drone equipped with the robotic arm}

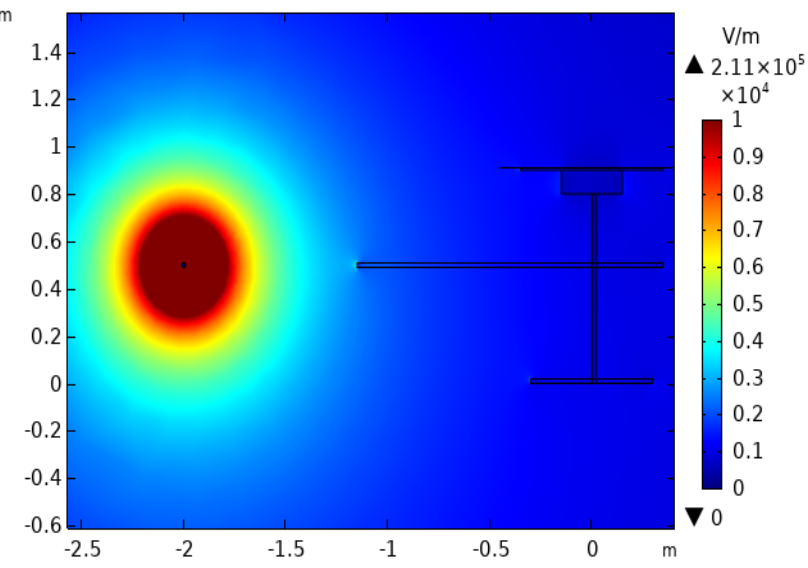

(a)

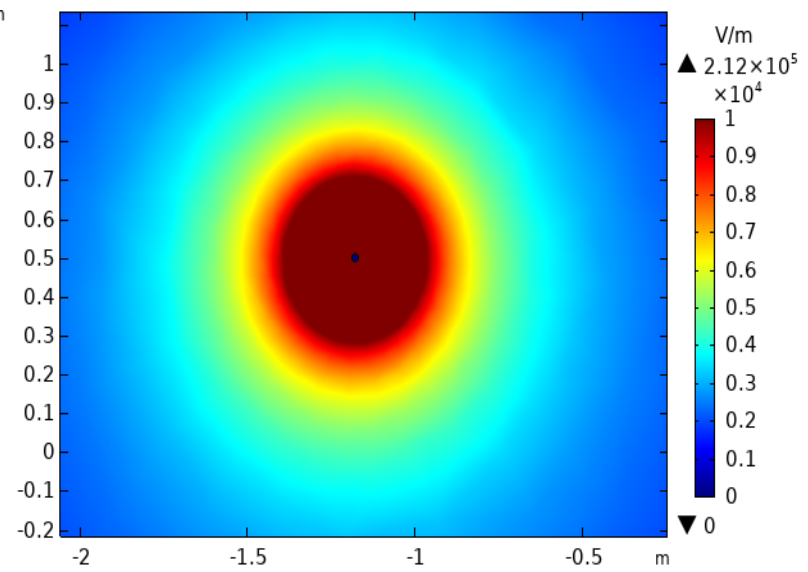

(b)

Fig3. Electric field distribution (a) $\mathrm{d}=0.84 \mathrm{~m}$, with inorganic human maintenance system (b) without UAV maintenance system.

Figure 3 (a) and (b) respectively show the electric field distribution of the maintenance system with or without a drone equipped with a robotic arm. By comparison, it can be seen that when the overhaul system of the UAV equipped with the robotic arm is $0.84 \mathrm{~m}$ away from the transmission line, the UAV overhaul system has almost no impact on the electric field around the transmission line.
However, it is obvious from figure 3(a) that the UAV inspection system has a significant increase in field strength at the left end of the robotic arm under the electric field. The electric field distribution in figure 3(a) shows the maximum electric field strength of each insulating component of the maintenance system of the UAV equipped with a robotic arm, as shown in table 1.

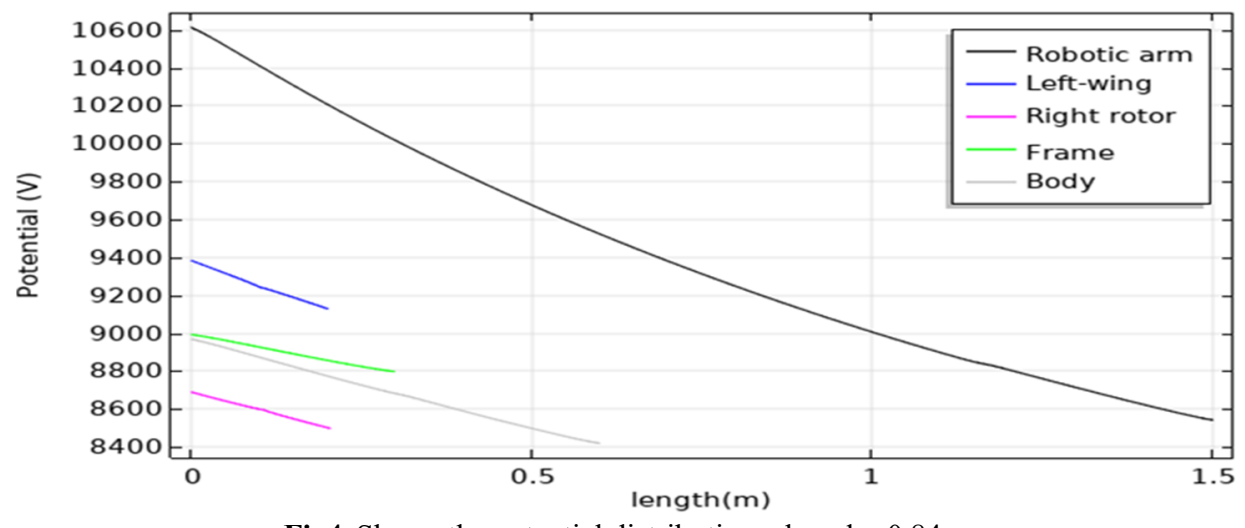

Fig4. Shows the potential distribution when $d=0.84 \mathrm{~m}$.

It can be seen from figure 4 that when $\mathrm{d}=0.84 \mathrm{~m}$, the electric potential distribution on the components of the drone maintenance system. According to figure 4, it can be seen that the electric potential of each component decreases from left to right as it is farther from the line. Taking into account that each insulating component has a certain withstand voltage capability, the voltage difference between the left and right ends of each component of the
UAV maintenance system can be obtained through the potential distribution on the components in figure 4 , as shown in Table 1 . It can be seen from table 1 that $\mathrm{d}=0.84 \mathrm{~m}$. Among the components of the maintenance system of the UAV equipped with a robotic arm, the electrical field strength of the robotic arm and the voltage difference between its two ends are the largest, which are $3712 \mathrm{v} / \mathrm{m}$ and $2069 \mathrm{v}$ respectively.

Table1. Maximum electric field strength and voltage when $\mathrm{d}=0.84 \mathrm{~m}$.

\begin{tabular}{lccccc}
\hline & Robotic arm & Left-wing & Right rotor & Frame & Body \\
\hline Field strength(V/m) & 3712 & 1838 & 1371 & 1730 & 720 \\
Voltage(V) & 2069 & 224 & 193 & 470 & 166 \\
\hline
\end{tabular}




\subsection{The distance between the overhaul system of the drone equipped with the robotic arm is $d=0.34 \mathrm{~m}$ from the transmission line}

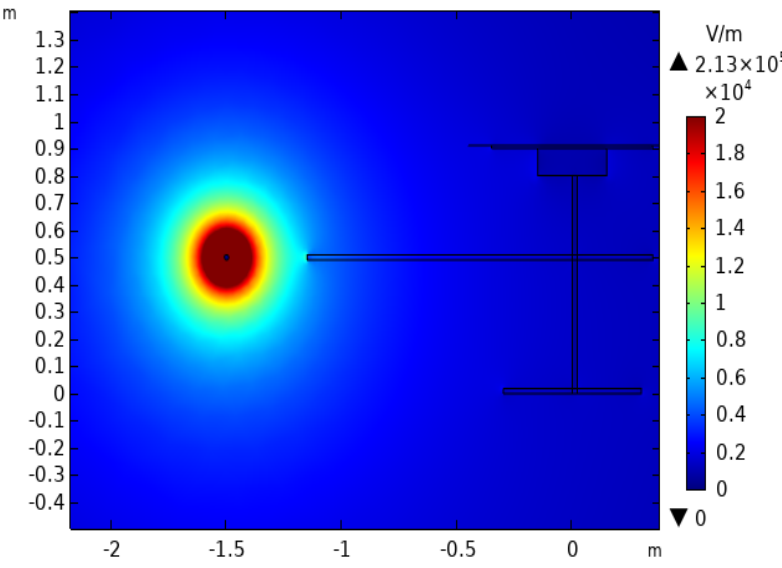

(a)

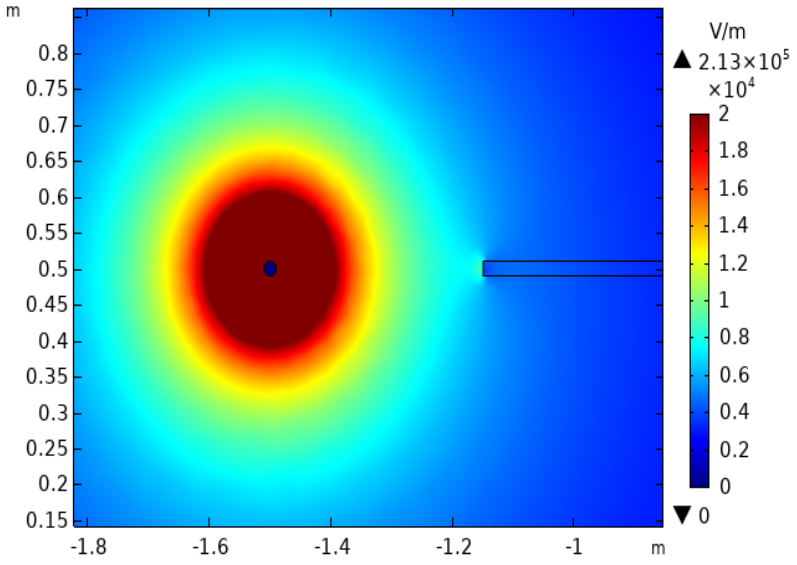

(b)

Fig5. Electric field distribution (a) system diagram (b) partial map.

Figure 3(a) and figure 5(a) are the electric field distribution of the system when $\mathrm{d}=0.84 \mathrm{~m}$ and $\mathrm{d}=0.34 \mathrm{~m}$, respectively. The comparison shows that the distance from the overhaul system of the drone equipped with the robotic arm is reduced to 0.34 from $0.84 \mathrm{~m}$ to the transmission line route. At $\mathrm{m}$, the influence of the field strength at the left end of the robotic arm of the UAV maintenance system becomes greater. It is obvious from figure $5(\mathrm{~b})$ that when the overhaul system of the drone equipped with the robotic arm is $0.34 \mathrm{~m}$ away from the transmission line, the electric field distribution of the transmission line at the left end of the robotic arm has slightly changed. Taking into account the risk of electrical discharge and breakdown of the insulating components of the UAV-equipped robotic arm maintenance system, the electric field strength of the components of the UAV maintenance system is shown in table 2 according to the electric field distribution in figure $5(\mathrm{a})$.

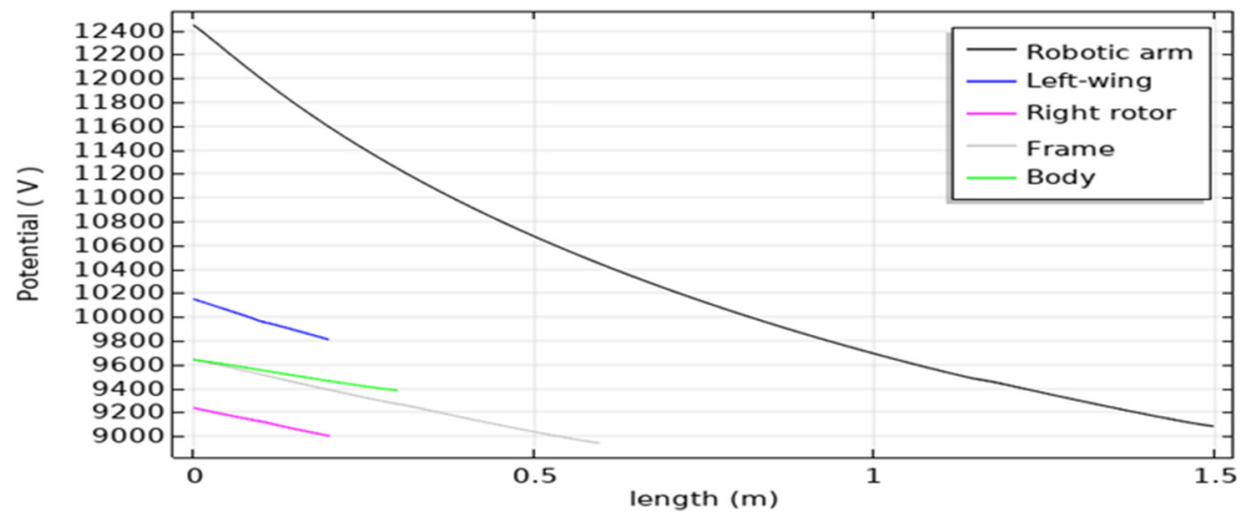

Fig6. Shows the potential distribution when $d=0.34 \mathrm{~m}$.

Figure 6 shows the potential distribution of the components of the UAV maintenance system when $\mathrm{d}=0.34 \mathrm{~m}$. Through the electric potential distribution on the components in figure 6 , the voltage difference across the components of the system is shown in table 1 .

Table2. Maximum electric field strength and voltage when $\mathrm{d}=0.34 \mathrm{~m}$.

\begin{tabular}{lccccc}
\hline & Robotic arm & Left-wing & Right rotor & Frame & Body \\
\hline Field strength(V/m) & 4656 & 2334 & 1260 & 1337 & 940 \\
Voltage(V) & 3353 & 338 & 236 & 700 & 229 \\
\hline
\end{tabular}




\subsection{The distance between the overhaul system of the drone equipped with the robotic arm is $\mathrm{d}=0.02 \mathrm{~m}$ from the transmission line}

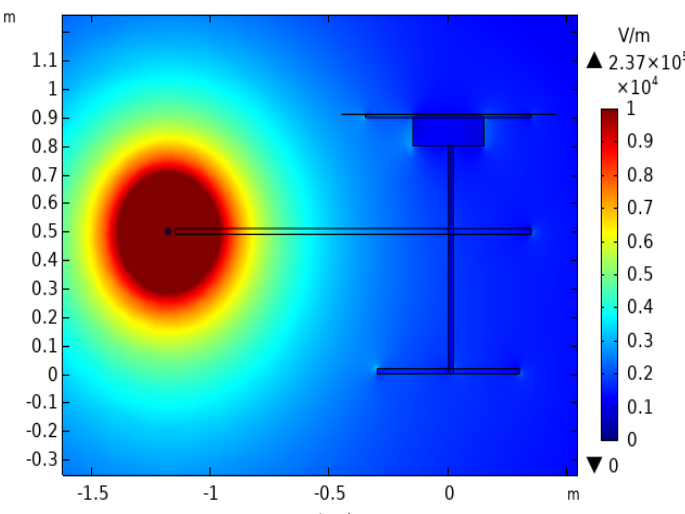

(a)

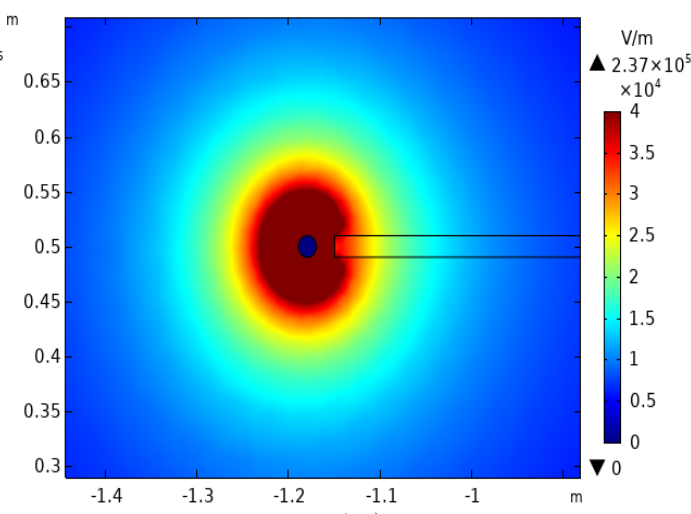

(b)

Fig7. Electric field distribution (a) system diagram (b) partial map.

Figure 7(a) is the electric field distribution diagram of the system when $\mathrm{d}=0.02 \mathrm{~m}$. $\mathrm{d}=0.02 \mathrm{~m}$ means that the dronemounted robotic arm maintenance system almost touches the transmission line. From figure 7(a), it can be seen that the electric field around the transmission line The UAV has the greatest impact on the overhaul system of the robotic arm. The edges of the robotic arm, frame, and fuselage under the UAV overhaul system have visible field strength increases. At the same time, see figure 7(b)
Comparing with figure 3(b), it can be clearly seen that the electric field distribution around the transmission line is changed under the influence of the UAV maintenance system. According to figure 7(a), the electric field intensity on each component of the UAV maintenance system is shown in table 3 . It can be seen from table 3 that when $\mathrm{d}=0.02 \mathrm{~m}$, the mechanical arm has the largest electric field strength compared to its insulating parts, which is $34673 \mathrm{v} / \mathrm{m}$.

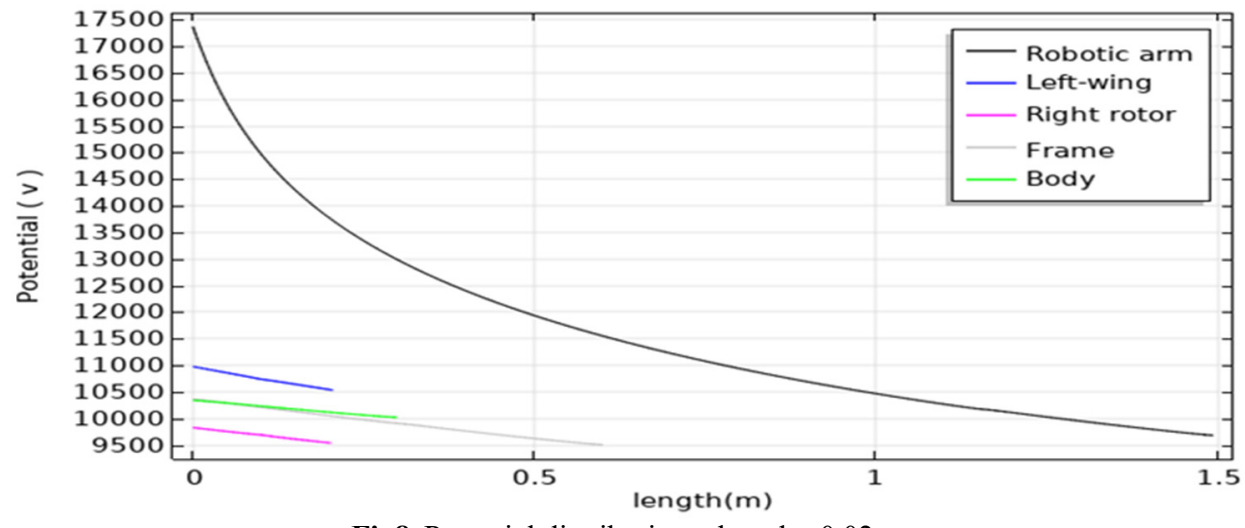

Fig8. Potential distribution when $\mathrm{d}=0.02 \mathrm{~m}$.

Figure 8 shows the potential distribution of each component when $\mathrm{d}=0.02 \mathrm{~m}$. According to the potential distribution, the voltage difference between the two ends of each component under the overhaul system based on the drone equipped with a robotic arm is obtained, as shown in table 3 . It is observed from table 3 that the voltage difference between the two ends of the fuselage is $298 V$. Compared with the voltage difference between the two ends of the fuselage in table 1 and table 2 , it is found that the voltage difference between the two ends of the fuselage is the largest at this time, that is, the withstand voltage is the highest at this time. . Since the airframe is closely connected with the communication system, in order to ensure the safe operation of the communication system, appropriate protective measures should be taken for the airframe.

Table3. Maximum electric field strength and voltage when $\mathrm{d}=0.02 \mathrm{~m}$.

\begin{tabular}{lccccc}
\hline & Robotic arm & Left-wing & Right rotor & Frame & Body \\
\hline Field strength(V/m) & 34363 & 2700 & 2193 & 2781 & 1179 \\
Voltage(V) & 7679 & 287 & 262 & 822 & 298 \\
\hline
\end{tabular}


Based on the above analysis, the influence of the electric field around the transmission line on the maintenance system of the drone equipped with a robotic arm is analyzed, and the research shows that the maintenance system of the drone equipped with a robotic arm is within the range of $0.02 \mathrm{~m}-0.84 \mathrm{~m}$ from the transmission line. The maximum electric field strength of each insulating component in the overhaul system of the robotic arm and the voltage difference between the left and right ends of each insulating component. From the comparative analysis of table 1 , table 2 and table 3 , it can be seen that the maximum electric field strength of each insulation component of the UAV maintenance system is less than the air breakdown strength $30 \mathrm{kV} / \mathrm{cm}[10]$, so when $\mathrm{d}$ is greater than or equal to $0.02 \mathrm{~m}$, each insulation component is always There is no discharge or breakdown. According to the simulation, it is concluded that the safe distance of the UAV equipped with the robotic arm to repair the transmission line is $0.02 \mathrm{~m}$. At the same time, in the range of $0.02 \mathrm{~m}-0.84 \mathrm{~m}$, the maximum voltage difference between the two ends of each insulating component is $7779 \mathrm{~V}$ for the robot arm, $338 \mathrm{~V}$ for the left rotor, $262 \mathrm{~V}$ for the right rotor, $298 \mathrm{~V}$ for the frame, and $822 \mathrm{~V}$ for the fuselage..

\section{Experimental Research}

In order to determine the minimum safe distance of the UAV equipped with a robotic arm for overhauling transmission lines, a test platform as shown in figure 9 was built on the basis of simulation. The test system mainly includes generators, voltage dividers, transformers, water resistance, and $1 \mathrm{~m}$ long transmission lines, UAV equipped with robotic arm overhaul system, etc., on this basis, carry out the safety distance test of UAV equipped with robotic arm overhauling transmission lines.

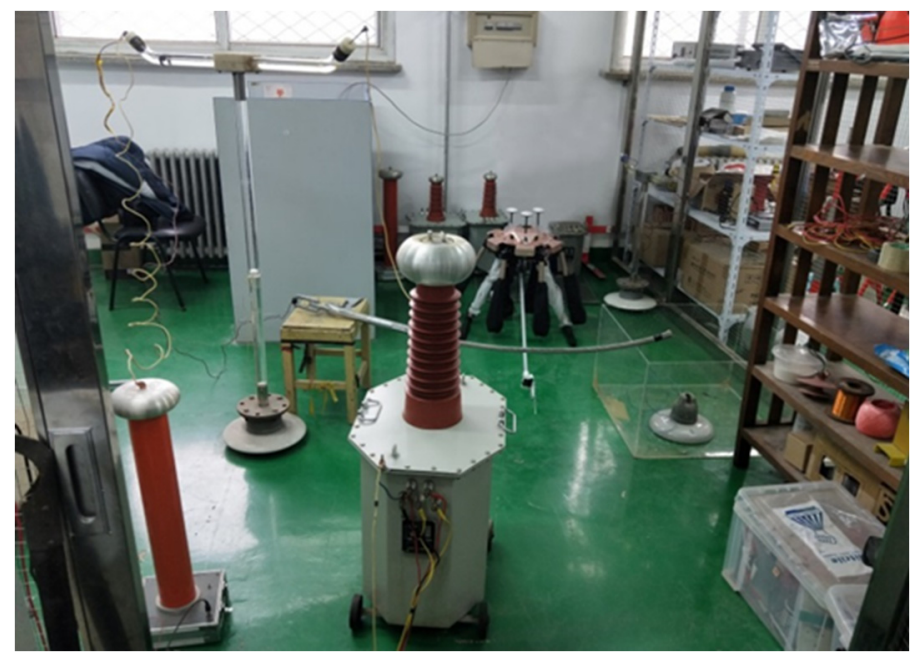

Fig9. Safety distance test platform

The drone used in the test is selected from DJI M600 PRO model, $1688 \mathrm{~mm}$ long, $1518 \mathrm{~mm}$ wide, and $727 \mathrm{~mm}$ high. Carbon fiber material, capable of long-term endurance, long-distance transmission, and broadcastlevel image transmission function; the robotic arm is $1.5 \mathrm{~m}$ long and has an inner diameter of $2 \mathrm{~cm}$. , The outer diameter is $2.5 \mathrm{~cm}$, using epoxy resin material with good insulation properties.

The test steps are as follows: (1) In the process of repairing power transmission lines with a known drone equipped with a robotic arm, the minimum distance between the left end of the robotic arm and the transmission line is $0.09 \mathrm{~m}$, and the drone used in the actual scene will be equipped with the robotic arm The overhaul system is placed steadily at the position where the minimum distance between the leftmost end of the robotic arm and the transmission line is $0.09 \mathrm{~m}$. (2) Charge the line on the test platform, adjust its phase voltage to $5 \mathrm{kV}, 10 \mathrm{kV}$, $15 \mathrm{kV}, 20.2 \mathrm{kV}$ in turn, move the robotic arm under the modulated phase voltage, and observe the insulation components of the robotic arm maintenance system on the drone Whether to discharge, and record the test phenomenon. (3) According to the test phenomenon, the minimum safe distance for repairing the power transmission line with the robot arm of the drone is obtained.

Test results: According to the safe distance test of the drone equipped with a robotic arm maintenance system, when the phase voltages of the built lines are $5 \mathrm{kV}, 10 \mathrm{kV}$, $15 \mathrm{kV}$, and $20.2 \mathrm{kV}$, the drone equipped with a robotic arm to repair the insulation components of the transmission line did not discharge And breakdown occurs, that is, the safety distance is $0.09 \mathrm{~m}$.

Comparing the test and simulation analysis: the test shows that in the actual application scenario, when the UAV is equipped with a robotic arm overhaul system to overhaul the transmission line, there is no discharge or breakdown of each insulating component, and the safety distance is $0.09 \mathrm{~m}$. Combined with the simulation, it is concluded that the minimum safe distance when the UAV is equipped with a robotic arm to repair the transmission line is $0.09 \mathrm{~m}$.

\section{Conclusion}

1) The electric field simulation was done on the power transmission line and the overhaul system of the UAV 
equipped manipulator, and the influence of the UAV overhaul system and the power transmission line was analyzed. The results show that, compared to the impact of the UAV maintenance system on the transmission line, the electric field around the transmission line has a greater impact on it.

2) It can be seen from the simulation that when the phase voltage of a certain phase of the transmission line is $35 / \sqrt{3} \mathrm{kV}$, the maximum voltage difference between the left and right ends of each insulating component is within the range of $0.02 \mathrm{~m}-0.84 \mathrm{~m}$ from the overhaul system of the robotic arm of the drone. They are: the robot arm is $7767 \mathrm{~V}$, the left rotor is $338 \mathrm{~V}$, the right rotor is $262 \mathrm{~V}$, the frame is $298 \mathrm{~V}$, and the fuselage is $822 \mathrm{~V}$.

3) Through the comparison of simulation and experiment, it can be seen that the minimum safe distance when the drone is equipped with a robotic arm to repair the transmission line is $0.09 \mathrm{~m}$, that is, the process of the drone equipped with a robotic arm to repair the transmission line is safe.

\section{Acknowledgement}

Foundation item: Science and Technology Service Project of State Grid Tianjin Electric Power Company in 2019(SGTJCN00YJJS1900527); Tianjin Science and Technology Plan Funding Project(18ZXZNGX00130)

\section{References}

1. Liu, G.S., Jia, J.Q. (2012) UAV applications and development in the power system[J]. Journal Of Northeast Dianli University., 32(1) : 53-36.

2. Lv, M., Luo, X., Liu, Z.Y. (2012) Unmanned helicopter study on the application of inspection and detection of power translation line[J]. HuBei Electric Power., 36(36) : 10-12.

3. Peng, X.Y., Qing, J.J., Mai, X.M., et al. (2016) Automatic power line inspection technology of large unmanned helicopter and its application[J]. Southern Power System Technology., 10(2): 24-31, 76.

4. Li, D., Lin, J.Y., GAO, P.Y., et al. (2016) Safe distance measurement for power transmission line inspection by UAVs[J]. Journal of Guangxi University( Nat Sci Ed)., 41(02) : 498-505.

5. Lv, M., Sheng, G.H., Zhang, W.D., et al. (2012) A tower and line tracking algorithm for power transmission line inspection based on unmanned aerial vehicles[J]. Automation of Electric Power Systems., 36(9) : 92-97.

6. Han, Z.X., Qiao, Y.H., et al. (2017) Research on image recognition based insulator fault detection method for UVA transmission line $[\mathrm{J}]$. Modern Electronics Technique., 40(22) : 179-181+186.

7. Jin, L.J., Wang, H., Wang, W.H., et al. (2018) Transmission line fault location based on machine vision and laser ranging[J]. Journal of Tongji University(Natural Science)., 46(12) : 1745-1753.

8. Mao, T.Q., Wang, C.C., Li, Y., et al. (2018)
Transmission line exaction method based on SGC stereo matching algorithm for UAV inspection image under complex backround[J]. Smart Power., 46(12) : 105-111.

9. Rong, Y.X., Wang, D.B. (2006) Application of Image Recognition Technology on Unmanned Aircraft's Auto - landing Navigation[J]. Computer Measurement \& Control., (08):1093-1095.

10. Liu, Z., Du, Y., Chen, Y., Ma, J.G., et al. (2019)Simulation and Experiment on the Safety Distance of Typical $\mu 500 \mathrm{kV}$ DC Transmission Lines and Towers for UAV Inspection[J].High Voltage Engineering., 45(02) : 426-432.

11. Wu J., Liu Z., Wu X.D., et al. (2017) Simulation Analysis of Safe Distance of Unmanned Aerial Vehicle in DC Transmission Line[J]. Hu Bei Electric Power., 41(04) : 14-19.

12. Fang, Y.Z., Liu, H.W., Liu, X., et al. (2019) Application of UAV Automatic Obstacle Avoidance Technology in the Inspection of Transmission Lines[J]. Shandong Electric Power., 46(02):10-13.

13. Shi S.S., Xie, L., Zhou, Z.B., Yang, H.M. (2019) Design of transmission line evading system based on UAV inspection[J]. Power Systems and Big Data., 22(08) : 67-74. 